- APPLIED PROBLEMS

- Published: 14 January 2021

\title{
Person Tracking and Reidentification for Multicamera Indoor Video Surveillance Systems
}

- $\underline{S . Y e}$,

- R.P. Bohush,

- H. Chen,

- I. Yu. Zakharava \&

- S. V. Ablameyko

\section{Pattern Recognition and Image}

Analysis volume 30, pages827-837 (2020)Cite this article

- 125 Accesses

- 2 Citations

- Metricsdetails

Abstract

For practical use, the relevance of indoor surveillance from multiple cameras to track the movement of people and reidentify them in video sequences is constantly increasing. This is a complex task due to the effect of uneven illumination, background inhomogeneity, overlap, uncertainty of the trajectories of people, and the similarity of their visual features. The article presents an approach to track people by video sequences and reidentify them in multicamera video surveillance systems that are used indoors. At the first step, people are detected using a YOLO v4 convolution neural network (CNN) and described by a rectangular area. Further, the search for the face area and the calculation of its features are carried out, which in the developed method are used when accompanying a person in a video sequence and during his intercamera reidentification. This approach improves the 
accuracy of tracking with a complex movement trajectory and multiple intersections of people with similar characteristics. The search for faces is carried out on the detected areas based on the multitasking MTCNN, and the MobileFaceNetwork model is used to form the vector of the features of the face. Human features are generated using a modified CNN based on ResNet34 and an HSV color tone channel histogram. The correspondence between people on different frames is established based on the analysis of the spatial coordinates of faces and people, as well as their CNN features, using the Hungarian algorithm. To ensure the accuracy of intercamera tracking, reidentification is performed based on the facial features. Five test video sequences of different numbers of people captured indoors with a fixed video camera were used to test and compare different approaches. The obtained experimental results confirmed the strength of the characteristics of the proposed approach.

This is a preview of subscription content, access via your institution.

\section{REFERENCES}

1. 1

P. W. Khan, Y.-Ch. Byun, and N. Park, "A data verification system for CCTV surveillance cameras using blockchain technology in smart cities," Electronics 9 (3), 484 (2020).

\section{Article Google Scholar}

2. 2

L. Duan, Y. Lou, Sh. Wang, W. Gao, and Y. Ru, "AI oriented large-scale video management for smart city: Technologies, standards, and beyond," IEEE MultiMedia 26 (2), 8- 20 (2019). 


\section{Article Google Scholar}

3. 3

R. Vezzani, D. Baltieri, and R. Cucchiara, "People reidentification in surveillance and forensics: A survey," ACM Comput. Surv. 46 (2), 1- 37 (2013).

\section{Article Google Scholar}

4.4

X. Wang, "Intelligent multi-camera video surveillance: A review," Pattern Recognit. Lett. 34 (1), 3- 19 (2013).

\section{Article Google Scholar}

5.5

R. P. Bohush and I. Y. Zakharava, "Person tracking algorithm based on convolutional neural network for indoor video surveillance," Comput. Opt. 40 (1), 109- 116 (2020).

\section{Article Google Scholar}

6. 6

D. Chahyati, M. I. Fanany, and A. M. Arymurthy, "Tracking people by detection using CNN features," in Proc. of the 4th Information Systems International Conference (ISICO 2017) (Bali, 2017), pp. 167- 172. 
N. Wojke, A. Bewley, and D. Paulus, "Simple online and realtime tracking with a deep association metric," in Proc. of the IEEE International Conference on Image Processing (ICIP2017) (Beijing, 2017), pp. 3645- 3649.

8.8

U. Iqbal, A. Milan, and J . Gall, "PoseTrack: J oint multiperson pose estimation and tracking," in Proc. of the IEEE Conference on Computer Vision and Pattern Recognition (CVPR2017) (Honolulu, HI, 2017), pp. 4654- 4663.

9.9

K. Koide, E. Menegatti, M. Carraro, M. Munaro, and J . Miura, "People tracking and re-identification by face recognition for RGB-D camera networks," in Proc. of the European Conference on Mobile Robots (ECMR) (Paris, 2017), pp. 1- 7.

10. 10

F. Schroff, D. Kalenichenko, and J . Philbin, "FaceNet: A unified embedding for face recognition and clustering," in Proc. of the IEEE Conference on Computer Vision and Pattern Recognition (CVPR) (Boston, Massachusetts, 2015), pp. 815- 823.

11. 11

R. Tao, E. Gavves, and A. W. M. Smeulders, "Siamese instance search for tracking," in Proc. of the IEEE Conference on Computer Vision and Pattern 
Recognition (CVPR) (Las Vegas, NV, 2016), pp. 14201429.

12. 12

L. Zhao, X. Li, Y. Zhuang, and J. Wang, "Deeply-learned part-aligned representations for person reidentification," in Proc. of the IEEE International Conference on Computer Vision (ICCV) (Venice, 2017), pp. 3239-3248.

13. 13

N. Narayan, N. Sankaran, D. Arpit, K. Dantu, S. Setlur, and V. Govindaraju, "Person re-identification for improved multi-person multi-camera tracking by continuous entity association," in Proc. of the IEEE Conference on Computer Vision and Pattern Recognition Workshops (CVPRW) (Honolulu, HI, 2017), pp. 64- 70 .

14. 14

A. Bochkovskiy, Ch.-Y. Wang, and H.-Y. M. Liao, YOLOv4: Optimal Speed and Accuracy of Object Detection (2020). https:/ / arxiv.org/ abs/ 2004.10934. Accessed J une 12, 2020.

15. 15

J . Deng, J . Guo, N. Xue, and S. Zafeiriou, ArcFace: Additive Angular Margin Loss for Deep Face Recognition (2019). https:// arxiv.org/ abs/ 1801.07698.pdf. Accessed May 20, 2020. 
16.

16

InsightFace Model Zoo.

https:/ / github.com/ deepinsight/ insightface/ wiki/ Model

-Zoo MTCNN. Accessed May 20, 2020.

17. 17

M. H. Ma and J. Wang, "Multi-view face detection and landmark localization based on MTCNN," in Proc. of the Chinese Automation Congress (CAC) (Xi'an, 2018), pp. 4200- 4205.

18. 18

Sh. Yang, P. Luo, Ch. Change Loy, and X. Tang, WIDER FACE: A Face Detection Benchmark (2015). https:/ / arxiv.org/ pdf/ 1511.06523.pdf. Accessed May 20, 2020.

19. 19

J . Deng, J . Guo, Y. Zhou, J . Yu, I. Kotsia, and S. Zafeiriou, RetinaFace: Single-stage Dense Face Localisation in the Wild (2019). https:/ / arxiv.org/ pdf/ 1905.00641.pdf. Accessed May 20, 2020.

20. 20

R. Bohush and I. Zakharava, "Robust person tracking algorithm based on Convolutional Neural Network for indoor video surveillance," in Pattern Recognition and Information Processing, PRIP 2019, Ed. by S. V. 
Ablameyko, V. V. Krasnoproshin, and M. M.

Lukashevich; Commun. Comput. Inf. Sci. 1055, 289300 (2019).

\section{21}

H. W. Kuhn, "The Hungarian method for the assignment problem," Naval Res. Logist. Q. 2, 83- 97 (1955).

\section{MathSciNet Article Google Scholar}

\section{22}

LabelImg Is a Graphical Image Annotation Tool and Label Object Bounding Boxes in Images. https:/ / github.com/ tzutalin/ labelImg. Accessed J une 16, 2019.

23. 23

B. Keni and R. Stiefelhagen, "Evaluating multiple object tracking performance: The CLEAR MOT metrics," EURASIP J . Image Video Process. 1, 1- 10 (2008).

\section{Google Scholar}

\section{Download references}

Funding

This work is supported by Public Welfare Technology Applied Research Program of Zhejiang Province (LGF19F020016, LGJ 18F020001 and LGJ 19F020002) and the National Highend Foreign Experts Program (GDW20183300463).

Author information 


\section{Zhejiang Shuren University, 310015, Hangzhou, China \\ S. Ye \& H. Chen}

2.Polotsk State University, 211440, Novopolotsk, Belarus

R. P. Bohush \& I. Yu. Zakharava

3. Belarusian State University, 220030, Minsk,

Belarus

S. V. Ablameyko

4. United Institute for Informatics Problems,

National Academy of Sciences of Belarus,

220012 , Minsk, Belarus

S. V. Ablameyko

Corresponding authors

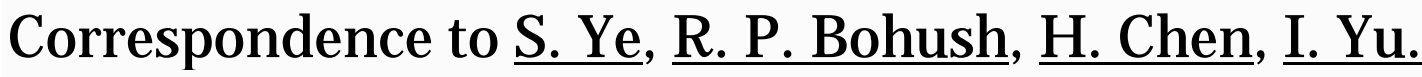
Zakharava or S. V. Ablameyko.

Ethics declarations

The authors declare that they have no conflict of interest.

Additional information

Shiping Ye. Born in 1967. Professor and Vice President of Zhejiang Shuren University. Graduated from Zhejiang University in 1988. Received master's degree in Computer Science and Technology from Zhejiang University in 2003. Scientific interests: application of computer graphics and images, GIS, machine learning. Author of more than 70 papers. Four research projects he has taken part in have been awarded the second prize of Zhejiang Provincial Scientific and Technological Achievement. Two teaching research programs he has presided over have been awarded first prize and second prize of Zhejiang Provincial Teaching Achievement. 
Rykhard Bohush. Graduated from Polotsk State University in 1997. Received doctoral degree in the field of Information Processing in 2002 at the Institute of Engineering Cybernetics, the National Academy of Sciences of Belarus. Head of Computer Systems and Networks Department of Polotsk State University. Scientific interests: image and video processing, object representation and recognition, intelligent systems, and machine learning.

Huafeng Chen. Born 1982. Associate Professor of Zhejiang Shuren University. Graduated from Zhejiang University in 2003. Received $\mathrm{PhD}$ in the field of Earth Exploration and Information Technology in 2009 at the Institute of Space Information and Technique, Zhejiang University. Scientific interests: remote sensing image processing, GIS application, image and video processing, and multiagent systems. Author of more than 10 papers.

Zakharava Iryna. Born 1995. Graduated from Polotsk State University in 2017. Received master of technical science degree in Mathematical Modeling, Numerical Methods, and Complexes of Programs in 2019. Studying for doctoral degree and working part-time as assistant in computer systems and networks department. Her scientific research is focused on image and video processing, scene understanding, and machine learning.

Sergey Ablameyko. Born in 1956. Received DipMath in 1978, doctoral degree in 1984, and doctor of science in 1990. Appointed professor in 1992 at Belarusian State University. Scientific interests: image analysis, pattern recognition, digital geometry, knowledge-based systems, geographical information systems, medical imaging. He is on the Editorial Board of Pattern Recognition Letters, Pattern Recognition 
and Image Analysis, and many other international and national journals. He is a senior member of IEEE, Fellow of IAPR, Fellow of Belarusian Engineering Academy, Academician of National Academy of Sciences of Belarus, Academician of the European Academy, and others. He was a First Vice-President of the International Association for Pattern Recognition (IAPR) (2006- 2008) and President of the Belarusian Association for Image Analysis and Recognition.

Rights and permissions

\section{$\underline{\text { Reprints and Permissions }}$}

About this article

Cite this article

Ye, S., Bohush, R.P., Chen, H. et al. Person Tracking and Reidentification for Multicamera Indoor Video Surveillance Systems. Pattern Recognit. Image Anal. 30, 827-837 (2020). https://doi.org/10.1134/S1054661820040136

Download citation

- Received23 June 2020

- Revised23 June 2020

- Accepted03 July 2020

- Published14 January 2021

- Issue DateOctober 2020

- DOI https://doi.org/10.1134/S1054661820040136 Keywords:

- tracking people

- face recognition

- internal video surveillance 
- convolution neural networks 